\title{
Towards improving usability requirements elicitation and specification using ontology- driven approach
}

\begin{abstract}
Nowadays, due to the high demand for quality software, the challenges facing by developers to meet the user expectations are increased from time to time. Usability, a quality attribute related to efficiency, effectiveness and satisfaction of the end users to a system has been recognized as one of the significant issue in software development. However, due to its abstract in characteristic which related to human behavior, usability requirements are always being ignored during the software development process especially from the early stage of requirement elicitation. As a result, it increases the cost of solving usability problems and affects the quality of final products. Therefore in this paper, we present a proposal to develop a pattern-based usability requirement elicitation and specification method by using ontology and boilerplates. The main contribution of this proposed work is to provide a guidance to help the requirement engineer to elicit and select the necessary usability features from the ontology knowledge repository and specifying them using boilerplate requirement template. Accordingly, a set of consistent, complete and unambiguous usability requirement specifications is produced.
\end{abstract}

Keyword: Boilerplates; Human computer interaction; Ontology; Requirement engineering; Usability eatterns 